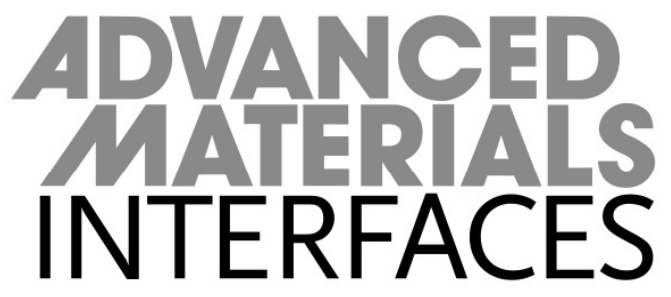

Postfach 101161 69451 Weinheim

Germany

WILEY-VCH

Courier services:

Boschstraße 12

69469 Weinheim

Germany

Tel.: (+49) 6201606235

Fax: (+49) 6201606500

E-mail: advmatinterfaces@wiley.com

Dear Author,

Please correct your galley proofs carefully and return them no more than four days after the page proofs have been received.

Please limit corrections to errors already in the text; cost incurred for any further changes or additions will be charged to the author, unless such changes have been agreed upon by the editor.

The editors reserve the right to publish your article without your corrections if the proofs do not arrive in time.

Note that the author is liable for damages arising from incorrect statements, including misprints.

Please note any queries that require your attention. These are indicated with a $Q$ in the PDF and a question at the end of the document.

Reprints may be ordered by filling out the accompanying form.

Return the reprint order form by fax or by e-mail with the corrected proofs, to WileyVCH : advmatinterfaces@wiley.com
To avoid commonly occurring errors, please ensure that the following important items are correct in your proofs (please note that once your article is published online, no further corrections can be made):

- Names of all authors present and spelled correctly

- Titles of authors correct (Prof. or Dr. only: please note, Prof. Dr. is not used in the journals)

- Addresses and postcodes correct

- E-mail address of corresponding author correct (current email address)

- Funding bodies included and grant numbers accurate

- Title of article OK

- All figures included

- Equations correct (symbols and sub/superscripts)

Corrections should be made directly in the PDF file using the PDF annotation tools. If you have questions about this, please contact the editorial office. The corrected PDF and any accompanying files should be uploaded to the journal's Editorial Manager site. 


\section{Author Query Form}

Journal

ADMI

Article

admi201901328

Dear Author,

During the copyediting of your manuscript the following queries arose.

Please refer to the query reference callout numbers in the page proofs and respond to each by marking the necessary comments using the PDF annotation tools.

Please remember illegible or unclear comments and corrections may delay publication.

Many thanks for your assistance.

\begin{tabular}{|c|c|c|}
\hline Query No. & Description & Remarks \\
\hline Q1 & $\begin{array}{l}\text { Please confirm that forenames/given names (blue) and surnames/family names } \\
\text { (vermilion) have been identified correctly. }\end{array}$ & $=$ \\
\hline Q2 & $\begin{array}{l}\text { Please provide the highest academic title (either Dr. or Prof.) for all authors, where } \\
\text { applicable. }\end{array}$ & $=$ \\
\hline Q3 & $\begin{array}{l}\text { Open access publication of this work is possible via Wiley OnlineOpen. Information } \\
\text { about OnlineOpen, the article publication charges (APCs), and Wiley's agreements with } \\
\text { various institutions to cover the APCs for their affiliated authors can be found at: https:// } \\
\text { authorservices.wiley.com/author-resources/Journal-Authors/licensing-open-access/open- } \\
\text { access/onlineopen.html. } \\
\text { The cost of publishing your manuscript OnlineOpen may be covered by one of Wiley's } \\
\text { national agreements. To find out more, log in to your Wiley Author Services account or } \\
\text { check the website: https://authorservices.wiley.com/author-resources/Journal-Authors/ } \\
\text { open-access/affiliation-policies-payments/index.html. Eligibility for these funds is based } \\
\text { on the affiliation of the primary corresponding author. }\end{array}$ & \\
\hline Q4 & Please define acronyms XRD and RHE at their first occurrence in the text. & $=$ \\
\hline Q5 & Please check the phrase ' It is thus reasonable to assume that the....' for correctness. & $=$ \\
\hline Q6 & Please provide the significance of ' $\mathrm{Pr}$ ' in name M. Barsoum. & $=$ \\
\hline Q7 & Please verify the publisher and publishers location in ref. [11]. & $=$ \\
\hline Q8 & Please provide the month of accession in ref. [30]. & \\
\hline Q9 & Please verify the additional details in ref. [31]. & 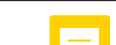 \\
\hline
\end{tabular}

Please confirm that Funding Information has been identified correctly.

Please confirm that the funding sponsor list below was correctly extracted from your article: that it includes all funders and that the text has been matched to the correct FundRef Registry organization names. If a name was not found in the FundRef registry, it may not be the canonical name form, it may be a program name rather than an organization name, or it may be an organization not yet included in FundRef Registry. If you know of another name form or a parent organization name for a "not found" item on this list below, please share that information.

\begin{tabular}{|l|l|}
\hline FundRef Name & FundRef Organization Name \\
\hline Agence National de la Recherche & \\
\hline European Union & European Commission \\
\hline Région Nouvelle Aquitaine & \\
\hline
\end{tabular}




\section{FULL PAPERS}

M. Benchakar, T. Bilyk, C. Garnero,

L. Loupias, C. Morais, J. Pacaud,

C. Canaff, P. Chartier, S. Morisset,

N. Guignard, V. Mauchamp, *

S. Célérier,* A. Habrioux*

1901328

MXene Supported Cobalt Layered Double Hydroxide Nanocrystals: Facile Synthesis Route for a Synergistic Oxygen Evolution Reaction Electrocatalyst

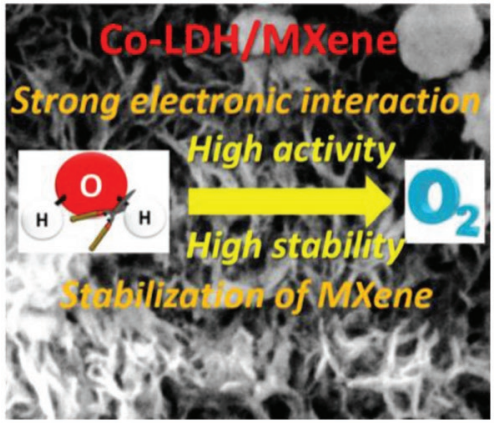

An innovative and facile synthesis route to obtain Co-based layered double hydroxide supported onto preserved MXene surface as highly efficient and stable oxygen evolution reaction electrocatalyst is described. The activity and stability of catalyst can be explained by a strong hydroxide/MXene electronic interaction. 


\title{
MXene Supported Cobalt Layered Double Hydroxide Nanocrystals: Facile Synthesis Route for a Synergistic Oxygen Evolution Reaction Electrocatalyst
}

\author{
Mohamed Benchakar, Thomas Bilyk, Cyril Garnero, Lola Loupias, Claudia Morais, \\ Jérôme Pacaud, Christine Canaff, Patrick Chartier, Sophie Morisset, Nadia Guignard, \\ Vincent Mauchamp, * Stéphane Célérier,* and Aurélien Habrioux**
}

The development of reliable electrolyzers is closely related to the development of a cost-effective highly active and stable electrocatalysts for the oxygen evolution reaction (OER). Herein, a simple method is used to synthesize a non-noble metal-based electrocatalyst for OER by synergistically coupling a catalytically active cobalt layered double hydroxide (Co-LDH) with a highly electrically conducting $2 \mathrm{D}$ transition metal carbide, $\mathrm{Ti}_{3} \mathrm{C}_{2} \mathrm{~T}_{x} \mathrm{MXene}$. The synergy between these two bidimensional materials (Co-LDH and $\mathrm{Ti}_{3} \mathrm{C}_{2} \mathrm{~T}_{x}$ ), evidenced by coupling electron energy loss spectroscopy and density functional theory simulations, results in superior electrocatalytic properties and makes possible having an excellent and stable oxygen evolution electrocatalyst. Moreover, the oxidative-sensitive MXene structure is preserved during the synthesis of the composite and the formation of a well recovering Co-LDH phase avoids the irreversible oxidation of MXene at high potential values, which may affect its conductivity. With an overpotential of $\approx 330 \mathrm{mV}$ at a current density of $10 \mathrm{~mA} \mathrm{~cm}^{-2}$ the catalyst exhibits a higher catalytic activity toward OER than commercial $\mathrm{IrO}_{2}$ catalysts.

\section{Introduction}

The sluggish kinetics of oxygen evolution reaction $(\mathrm{OER})^{[1]}$ currently hampers the large scale development of many energy storage and conversion devices such as metal-air batteries or water electrolyzers required to implement the future energy

M. Benchakar, Dr. C. Garnero, L. Loupias, Dr. C. Morais, C. Canaff,

S. Morisset, N. Guignard, Dr. S. Célérier, Dr. A. Habrioux

Institut de Chimie des Milieux et Matériaux de Poitiers (IC2MP)

Université de Poitiers

CNRS

F-86073 Poitiers, France

E-mail: stephane.celerier@univ-poitiers.fr;

aurelien.habrioux@univ-poitiers.fr

T. Bilyk, Dr. C. Garnero, Dr. J. Pacaud, Dr. P. Chartier, Dr. V. Mauchamp Institut Pprime

UPR 3346 CNRS

Université de Poitiers

ISAE-ENSMA

BP 30179, 86962 Futuroscope-Chasseneuil Cedex, France

E-mail: vincent.mauchamp@univ-poitiers.fr

The ORCID identification number(s) for the author(s) of this article can be found under https://doi.org/10.1002/admi.201901328.

DOI: 10.1002/admi.201901328
$\mathrm{LDH}$ can be described by the general formula 37 $\left[\mathrm{M}_{1-x}^{2+} \mathrm{M}_{x}^{3+}(\mathrm{OH})_{2}\right]^{x+}\left[\mathrm{A}_{x / n}\right]^{n-}, \quad m \mathrm{H}_{2} \mathrm{O}$ where $\mathrm{M}^{2+}$ and $\mathrm{M}^{3+}$ are 38 respectively divalent and trivalent cations and $\mathrm{A}^{n-}$ is the inter- 39 layer anion. ${ }^{[6 \mathrm{~d}]}$ The LDH structure is based on the partial 40 exchange of divalent and trivalent metal cations in the main 41 layer. The positive charge of the main layer is compensated 42 by the intercalation of anions in the interlayer spacing. LDH 43 materials, however, exhibit two major drawbacks. First, the pro- 44 posed synthesis routes are complex and involve either the ex 45 situ preparation of zeolitic imidazolate framework-67 (ZIF-67) ${ }^{[7]} 46$ or its in situ formation. ${ }^{[6 c]}$ Second, these materials suffer from 47 a poor intrinsic electronic conductivity. To overcome the latter, 48 LDH can be strongly coupled to conductive materials (carbon 49 nanotubes CNT, reduced graphene oxide rGO, etc.) with the 50 aim to make a highly conductive matrix with engineered het- 51 erointerfaces capable of promoting fast charge transfer kinetics 52 during OER. ${ }^{[8]}$

For this purpose, MXenes nitrides or carbides, ${ }^{[9]}$ have 54 recently attracted much attention because of their high elec- 55 tronic conductivity $\left(8000-10000 \mathrm{~S} \mathrm{~cm}^{-1}\right) \cdot{ }^{[10]}$ MXenes consist of 56 $\mathrm{M}_{n+1} \mathrm{X}_{n}$ octahedra layers ( $n=1$ to 3 ), which are obtained by the 57 exfoliation of the A element from $\mathrm{M}_{n+1} \mathrm{AX}_{n}$ precursors, a family 58 of 70+ known ternary carbides and nitrides, the so-called MAX 59 

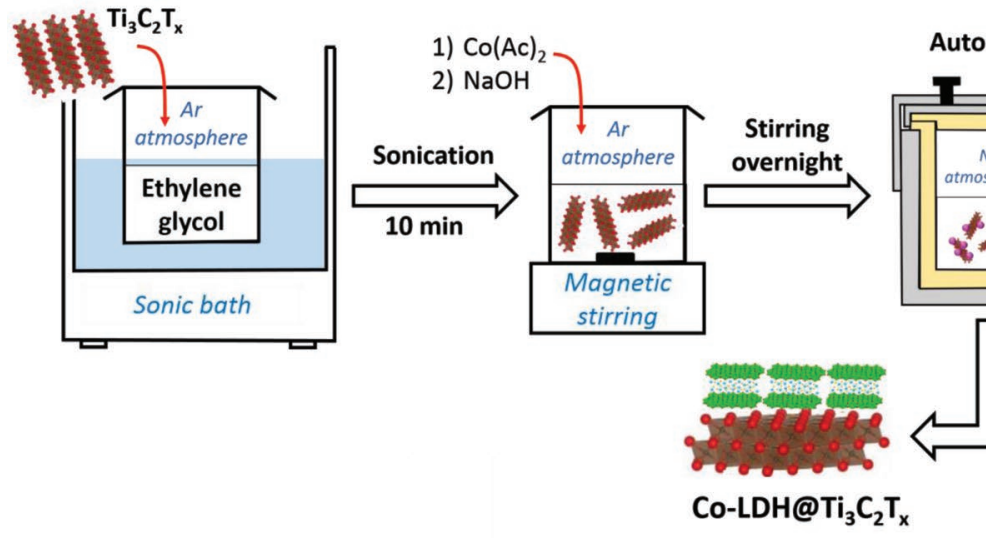

Scheme 1. Schematic view of the protocol followed to synthesize Co-LDH@Ti ${ }_{3} C_{2} T_{x}$ material.

phases ( $\mathrm{M}=$ transition metal, A: group III-A or IV-A elements, $\mathrm{X}$ : C and/or N) $\cdot^{[11]}$ Moreover, the surface of MXenes sheets is covered with terminal groups $(\mathrm{T}=-\mathrm{OH},-\mathrm{O}$, and/or $-\mathrm{F})$ chemically bonded to the metal atoms. These different $\mathrm{T}$ terminal groups of $\mathrm{M}_{n+1} \mathrm{X}_{n} \mathrm{~T}_{x}$ are formed during the exfoliation step and their nature $(\mathrm{OH} / \mathrm{O} / \mathrm{F}$ ratio) depends on the exfoliation environment. ${ }^{[12]}$ They can also act as anchoring points for nanostructured active phase, thus enabling a high dispersion degree of nanocatalysts. In addition, the electron density on the catalyst surface atoms can be altered by the formation of MXene/ active phase heterointerfaces, which is clearly of upmost importance in the field of catalysis research. ${ }^{[5 a]}$

The wide range of possible substitutions in the $\mathrm{M}, \mathrm{X}$ (core), and $\mathrm{T}$ (surface) sites of MXenes leads to a large family of 2D $\mathrm{M}_{n+1} \mathrm{X}_{n} \mathrm{~T}_{x}$ materials with tunable properties opening an immense, and largely unexplored, field of potential applications. Among them, $\mathrm{Ti}_{3} \mathrm{C}_{2} \mathrm{~T}_{x}$ is the most studied material and several works using it as support of electrocatalyst for OER such as $\mathrm{C}_{3} \mathrm{~N}_{4},{ }^{[13]} \mathrm{MOF},{ }^{[14]} \mathrm{NiCoS},{ }^{[15]} \mathrm{CoP},{ }^{[16]} \mathrm{Co} / \mathrm{N}-\mathrm{CNT},{ }^{[17]}$ cobalt borate, ${ }^{[18]} \mathrm{FeOOH}$ quantum dots, ${ }^{[19]}$ black phosphorus quantum dots, ${ }^{[20]}$ and FeNi-LDH ${ }^{[8 b]}$ have shown very promising performances. Nevertheless, $\mathrm{Ti}_{3} \mathrm{C}_{2} \mathrm{~T}_{x}$ MXene is prone to irreversible surface oxidation at $\approx 0.45 \mathrm{~V}$ versus $\mathrm{Ag} / \mathrm{AgCl}$, ${ }^{[21]}$ leading to the formation of $\mathrm{TiO}_{2}$ clusters. This strongly modifies its surface electronic properties in strong alkaline media and at electrode potentials required for the OER. As a consequence the stabilization of MXene in this potential range is a major challenge. Unfortunately, in numerous reported studies on their use for oxygen electrocatalysis no information concerning the surface chemical state of MXene under operating conditions is provided and no special attention is paid to their potential surface oxidation.

Herein, we report an innovative and quite simple polyol synthesis method enabling heterogeneous nucleation and growth of cobalt layered double hydroxides Co-LDH on the surface of $\mathrm{Ti}_{3} \mathrm{C}_{2} \mathrm{~T}_{x}$ sheets without using the ZIF-67 chemical agent. We show, for the first time that using this synthesis route, the MXene surface state can be protected from oxidation. Numerous physico-chemical characterization techniques were employed in order to establish structural, morphological, and electronic properties of the obtained composite material exhibiting promising catalytic properties for the OER in alkaline media. Special attention was paid to the examination of interaction between Co-LDH and $\mathrm{Ti}_{3} \mathrm{C}_{2} \mathrm{~T}_{x}$ and to the surface chemical state of the MXene substrate. It is shown that the heterogeneous nucleation of Co-LDH phase results in the modification of the $\mathrm{Ti}_{3} \mathrm{C}_{2} \mathrm{~T}$ surface's electronic structure. This study constitutes therefore a major contribution as it paves the way for the design of a new class of materials for OER involving a recently discovered and very large family of 2D supports.

\section{Results and Discussion}

\subsection{Composite Characterization}

The facile synthesis route used to obtain Co-LDH@ $\mathrm{Ti}_{3} \mathrm{C}_{2} \mathrm{~T}_{x}$ material is detailed in Scheme 1. It is a quite simple two-step polyol process. After adsorption of reactants on MXene surface, the formation of Co-LDH phase at MXene surface occurs consequently to a solvothermal treatment as described in the Experimental Section.

The XRI atterns of $\mathrm{Ti}_{3} \mathrm{C}_{2} \mathrm{~T}_{x}, \mathrm{Co}-\mathrm{LDH} @ \mathrm{Ti}_{3} \mathrm{C}_{2} \mathrm{~T}_{x}$, and Co-LDH, recu...d with a Co $\mathrm{K}_{\alpha}$ radiation source are reported in Figure 1.

As evidenced by the absence of sharp diffraction peaks at $2 \theta$ values of $11.1^{\circ}$ and $22.3^{\circ}$ (see MAX XRD pattern-Figure S1 in the Supporting Information) in the diffraction pattern of the $\mathrm{Ti}_{3} \mathrm{C}_{2} \mathrm{~T}_{x}$ sample (Figure 1a), the initial $\mathrm{Ti}_{3} \mathrm{AlC}_{2}$ MAX phase has been fully converted into MXene. According to inductively coupled plasma optical emission spectroscopy (ICP-OES) analysis, a very small amount of $\mathrm{Al}$ (lower than $0.05 \mathrm{Al}$ per $\mathrm{Ti}_{3} \mathrm{C}_{2}$ unit) remains in the sample, confirming $\mathrm{Al}$ etching. The $c$ parameter calculated for $\mathrm{Ti}_{3} \mathrm{C}_{2} \mathrm{~T}_{x}$ sample is of $22.6 \AA$, which is in good agreement with values reported for samples prepared using the $\mathrm{LiF} / \mathrm{HCl}$ etching method and dried at $80^{\circ} \mathrm{C} \cdot{ }^{[12 c]}$ Shoulders affecting the symmetry of 001 diffraction lines (e.g., (002) line at $\approx 9^{\circ}$ or (004) line at $\approx 19.5^{\circ}$ ) can moreover be observed. These can be ascribed to an interstratification phenomenon often encountered on these claylike materials and indicating the coexistence of structures having different layer-to-layer distances. ${ }^{[22]}$

The Co-LDH sample (Figure 1c) is composed of a mixture of several phases: Co-LDH with characteristic peaks at $13.57^{\circ}(003)$, $27.32^{\circ}(006) 39.57^{\circ}(010), 40.43^{\circ}(012), 54.89^{\circ}(0012)$, and $71.26^{\circ}$ 


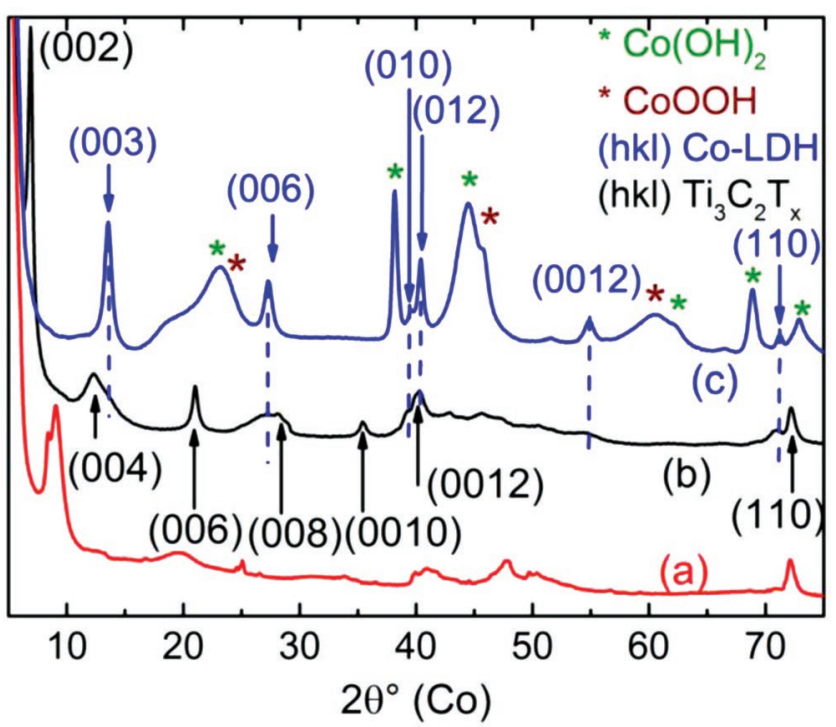

Figure 1. XRD patterns of a) $\mathrm{Ti}_{3} \mathrm{C}_{2} \mathrm{~T}_{x}$, b) Co-LDH @ $\mathrm{Ti}_{3} \mathrm{C}_{2} \mathrm{~T}_{x}$, and c) Co-LDH. All diffractograms were recorded with a $\mathrm{Co}_{\alpha}$ radiation source to limit fluorescence.

(110) (close to the JCPDS file no-00-046-0605 of $\mathrm{Co}_{5}\left(\mathrm{O}_{9.48} \mathrm{H}_{8.52}\right)$ $\mathrm{NO}_{3}$ layered double hydroxides), $\mathrm{Co}(\mathrm{OH})_{2}$ (JCPDS file no-00030-0443), and $\mathrm{CoOOH}$ (JCPDS file no-01-073-0497). Taking into account that no nitrates are used in our synthesis, the intercalated anions between Co-LDH layers are acetate ions from the cobalt precursor salt. This intercalation process does not significantly impact the interlayer spacing since the 001 reflection peaks positions are close to those reported for $\mathrm{Co}_{5}\left(\mathrm{O}_{9.48} \mathrm{H}_{8.52}\right)$ $\mathrm{NO}_{3}$ material. On the diffraction patterns recorded for Co-LDH@ $@ \mathrm{Ti}_{3} \mathrm{C}_{2} \mathrm{~T}_{x}$ composite (Figure 1b), (001) and (110) diffraction lines of the MXene phase are clearly observed indicating the good preservation of this oxidation-sensitive phase during the composite synthesis by our newly developed polyol method. No diffraction lines typical of $\mathrm{TiO}_{2}$ can be observed, further confirming that bulk oxidation of MXene does not occur. However, a dramatic shift of the (002) peak toward lower diffraction angles leading to a c parameter value of $29.7 \AA$ can be noted in comparison with the initial MXene phase $(c=22.6 \AA)$. This shift is higher than the one observed for MXene obtained using Li salts as etching agent and dried at ambient conditions (around 24-25 $\AA)^{[22]}$ and can be explained by the insertion of cobalt and water molecules between the MXene sheets. The possibility to insert 3d transition metal cations such as $\mathrm{Fe}^{n+}$ or $\mathrm{Co}^{n+}$ between MXene sheets has already been demonstrated in our previous works. ${ }^{[12 c]}$ At first sight, the diffraction pattern given in Figure $1 \mathrm{~b}$ does not give any clear evidence of the formation of a well-crystallized cobalt-based phase. In the $2 \Theta$ range where no intense diffraction lines associated to the LDH phase (i.e., at diffraction angles lower than $10^{\circ}$, between $15^{\circ}$, and $25^{\circ}$ and between $30^{\circ}$ and $39^{\circ}$ ), diffraction lines corresponding to MXene phase are sharper ((002), (006), and (0010) diffraction lines for example). Nevertheless, one can notice that some reflection lines ascribed to the MXene phase ((004) and (008) diffraction lines for example) are quite broad. This shows the presence of an additional contribution superimposed to the
MXene diffraction lines, which could correspond to (003) and 1 (006) reflection lines of Co-LDH. Moreover the (110) reflec- 2 tion line $\left(72^{\circ}\right.$, Figure $\left.1 \mathrm{~b}\right)$ of the Co-LDH phase is also observed 3 in the diffraction pattern recorded for the composite material 4 while it is not the case for MXene sample, definitely attesting 5 to the presence of crystallized LDH in this sample. Contrarily 6 to the pattern recorded with unsupported Co-based material 7 (Figure 1c), $\mathrm{Co}(\mathrm{OH})_{2}$ and $\mathrm{CoOOH}$ crystallized phases are not 8 observed in the composite suggesting that the MXene promotes 9 the formation of LDH. This is probably due to the presence of 10 terminal groups such as $-\mathrm{F},-\mathrm{OH}$, and $-\mathrm{O}$, which may favor the 11 nucleation of Co-LDH as already reported during the synthesis 12 of NiFe-LDH/MXene material. ${ }^{[8 b]}$

To confirm the formation of the Co-LDH phase with organic 14 counter ions, electron energy loss spectroscopy (EELS) as well 15 as energy filtered electron diffraction (EFED) were performed 16 on Co-LDH@ $\mathrm{Ti}_{3} \mathrm{C}_{2} \mathrm{~T}_{x}$ composite in a Co-rich region. Given that 17 Co-LDH material is highly beam sensitive, ${ }^{[6]]}$ great care was 18 taken to study its irradiation induced damages during transmis- 19 sion electron microscopy (TEM) observations. The evolution of 20 the sample morphostructural and electronic properties through 21 EFED and EELS were then examined upon electron beam irra- 22 diation (see Figure S2 and associated comments in part II in 23 the Supporting Information). An identification of the phase 24 before irradiation (Figure 2a) was then attempted. 25

Focusing first on the EDP, two diffuse rings are visible 26 (Figure 2a): the interplanar distances calculated from these two 27 rings are 2.67 and $1.57 \AA$. The here-measured distances are in 28 good agreement with those reported for Co-LDH by $\mathrm{Xu}$ et al. 29 using a similar TEM analysis: $d_{012}{ }^{\mathrm{LDH}}=2.69 \AA$ and $d_{110}{ }^{\mathrm{LDH}}=30$ $1.56 \AA .{ }^{[6 c]}$ Moreover, they are in good agreement with the 31 $d_{012}{ }^{\mathrm{LDH}}=2.60 \AA$ and $d_{110}{ }^{\mathrm{LDH}}=1.54 \AA$ determined from XRD 32 (Figure 1) confirming the formation of the Co-LDH phase. A 33 typical carbon $\mathrm{K}$ edge recorded in the Co-LDH phase is pre- 34 sented in Figure $2 \mathrm{~b}$ (bottom part). The shape of the C K-edge 35 strongly differs from that of the MXene (top part of Figure 2b). 36 In particular, the sharp peak at the edge onset (labeled A) is 37 consistent with molecular-like levels. ${ }^{[23]}$ It is thus reasonable to 38 assume that the here-evidenced molecular carbon corresponds 39 to the acetate counter-anions of the Co-LDH phase.

The Ti and Co contents of Co-LDH@ $\mathrm{Ti}_{3} \mathrm{C}_{2} \mathrm{~T}_{x}$ composite were measured by inductively coupled plasma optical emis- 42 sion spectroscopy. A Co/Ti atomic ratio of 1.4 was obtained, 43 which is close to the target content (ratio of 1.2). The small 44 difference can be explained by the initial MXene phase, which 45 is not exactly $\mathrm{Ti}_{3} \mathrm{C}_{2}(\mathrm{OH})_{2}$ because of the probable presence of 46 water between the sheets and the mixture of different terminal 47 groups. Moreover, the decanted water after centrifugation is 48 uncolored indicating that all initial introduced Co ions remain 49 in the composite indicating high covering rate of the MXene by 50 Co-LDH.

Scanning electron microscope (SEM) micrographs pre- 52 sented in Figure 3a,b clearly show the typical 2D-like MXene 53 morphology of the $\mathrm{Ti}_{3} \mathrm{C}_{2} \mathrm{~T}_{x}$ initial material. Sheets are tightly 54 stacked as reported for MXene synthesized with the etching 55 method involving the use of metal salts such as LiF. ${ }^{[12 b, c]}$ The 56 morphology of this kind of sample strongly differs from that of 57 MXenes obtained using HF as the etching agent. The reason 58 is that the harsh HF environment leads to more defects and 59 

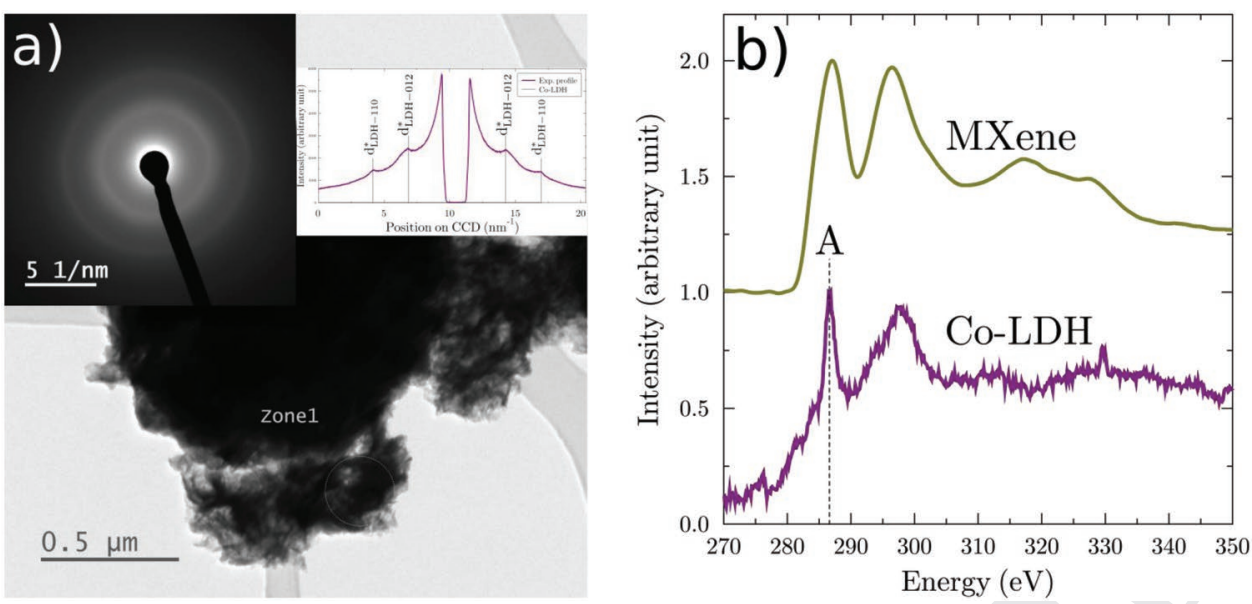

Figure 2. a) TEM micrography, together with the corresponding diffraction pattern and associated intensity profile. b) Comparison between typical $\mathrm{C}$ K-edges obtained on a reference $\mathrm{Ti}_{3} \mathrm{C}_{2} \mathrm{~T}_{x}$ flake and in the Co-LDH phase.

thus to well separated sheets. In contrast, the milder conditions associated to the $\mathrm{LiF} / \mathrm{HCl}$ environments together with the induced intercalation of cations (i.e., $\mathrm{Li}^{+}$in the present case), which stabilize water layers strengthen the cohesion between the sheets leading to more uniform structures as explained in ref. [12b] and [12c]. For Co-LDH@ $@ \mathrm{Ti}_{3} \mathrm{C}_{2} \mathrm{~T}_{x}$, at low magnification (Figure 3c,d), MXene multilayers seem to be embedded into a Co-LDH matrix composed of porous sphere-like agglomerates. At higher magnifications these individual spheres are composed of LDH nanosheets forming an interconnected porous network (Figure 3e,f). This microstructure is similar to those reported for other LDH phases. ${ }^{[6 c, 8 b]}$ The presence and relative uniform distribution at macroscopic scale of Ti (from MXene) and Co (from Co-LDH) elements in the Co-LDH@ $@ \mathrm{Ti}_{3} \mathrm{C}_{2} \mathrm{~T}_{x}$ composite is revealed by elemental mapping analysis (Figure S3, Supporting Information) even if one cannot exclude the presence of some Co-rich or Ti-rich areas.

The Raman spectra of the as-synthesized $\mathrm{Ti}_{3} \mathrm{C}_{2} \mathrm{~T}_{x}$ and Co-LDH@ $@ \mathrm{Ti}_{3} \mathrm{C}_{2} \mathrm{~T}_{x}$ composite are presented in Figure 4. For the MXene as well as the composite material, the spectra recorded present all the bands consistent with known vibrational modes of $\mathrm{Ti}_{3} \mathrm{C}_{2} \mathrm{~T}_{x} \cdot{ }^{[24]}$ This shows once again the preservation of the MXene during the composite synthesis. In addition, Raman bands centered at $\approx 458$ and $518 \mathrm{~cm}^{-1}$ were also observed and match with the position of the vibrational modes reported for pure Co-LDH ${ }^{[6 c]}$ indicating again the Co-LDH formation from our synthesis route.

The surface chemistry of the Co-LDH@ $@ \mathrm{Ti}_{3} \mathrm{C}_{2} \mathrm{~T}_{x}$ composite was also investigated by XPS (Figure 5) by recording signals associated to Co2p (Figure 5a), C1s (Figure 5c), and Ti2p (Figure S4, Supporting Information) core level spectra. The Co2p signal is quite similar to the one recorded with $\mathrm{Co}(\mathrm{OH})_{2}$ commercial powder (Figure $5 \mathrm{~b}$ ) attesting for the presence of $\mathrm{Co}^{2+}$ in the Co-LDH phase. No clear identification of $\mathrm{Co}^{3+}$ can be performed suggesting that Co-LDH contains a low amount of $\mathrm{Co}^{3+}$ cations.

In the C1s spectral region (Figure 5c), two major contributions are observed, centered at $\approx 287$ and $\approx 290 \mathrm{eV}$, and can be respectively ascribed to $\mathrm{C}=\mathrm{O}$ and $\mathrm{O}-\mathrm{C}=\mathrm{O}$ groups providing direct evidence for the presence of acetate ions as interlayer anions in the LDH material. The $\mathrm{Ti}-\mathrm{C}$ bond contribution to the $\mathrm{C} 1 \mathrm{~s}$ signal for as-synthesized $\mathrm{Ti}_{3} \mathrm{C}_{2} \mathrm{~T}_{x}$ is centered at $\approx 282 \mathrm{eV}$ (Figure $5 \mathrm{~d}$ ). This contribution can also be observed for the composite but the magnitude of the signal is quite low. This is explained by the low analysis depth of the XPS technique ( 5 to $10 \mathrm{~nm}$ ) and by the high coverage of the MXene phase with Co-LDH, as shown in SEM images (Figure 3). This is further confirmed by the very low global intensity of the Ti2p core level spectrum obtained for the composite as compared to the spectrum obtained for the $\mathrm{Ti}_{3} \mathrm{C}_{2} \mathrm{~T}_{x}$ MXene (Figure S4 and associated comments in part IV in the Supporting Information). Nevertheless, the presence of the different Ti2p contributions of the MXene in the composite again indicates the preservation of MXene surface state during the synthesis (see part IV in the Supporting Information).

\subsection{Interaction between Co-LDH and $\mathrm{Ti}_{3} \mathrm{C}_{2} \mathrm{~T}_{x}$ Phases}

A key point in the design of an efficient composite for electrocatalysis is the synergy between the different building blocks. This interaction, the characterization of which is very challenging and thus rarely successfully achieved, has been probed using an approach based on the combination of TEM-EELS and density functional theory (DFT) calculations. The results are presented in Figures 6, 7, and 8. Focusing first on Figure 6, one can observe that, on average, the MXene sheets keep their integrity when mixed with the nanoparticles during the formation of composites. Depending on the local Co-phase coverage, the stacking of the MXene sheets is more or less disorganized evidencing an intimate mixing between the MXene and Co-LDH electrocatalyst. As an illustration, Figure 6a evidences a clean MXene sheet (so-called zone 1-a) with sharp edges and homogeneous TEM contrast (confirmed by the diffraction pattern given in Figure 6b) in close contact with the fibrous Co-LDH (see zones 1b-green circles). In other regions, as evidenced in Figure $6 c$ (corresponding to zone 2), the MXene stack is structurally more perturbed as revealed by the inhomogeneous contrasts and a more irregular shape. The corresponding diffraction pattern, presented in Figure $6 \mathrm{~d}$, is 

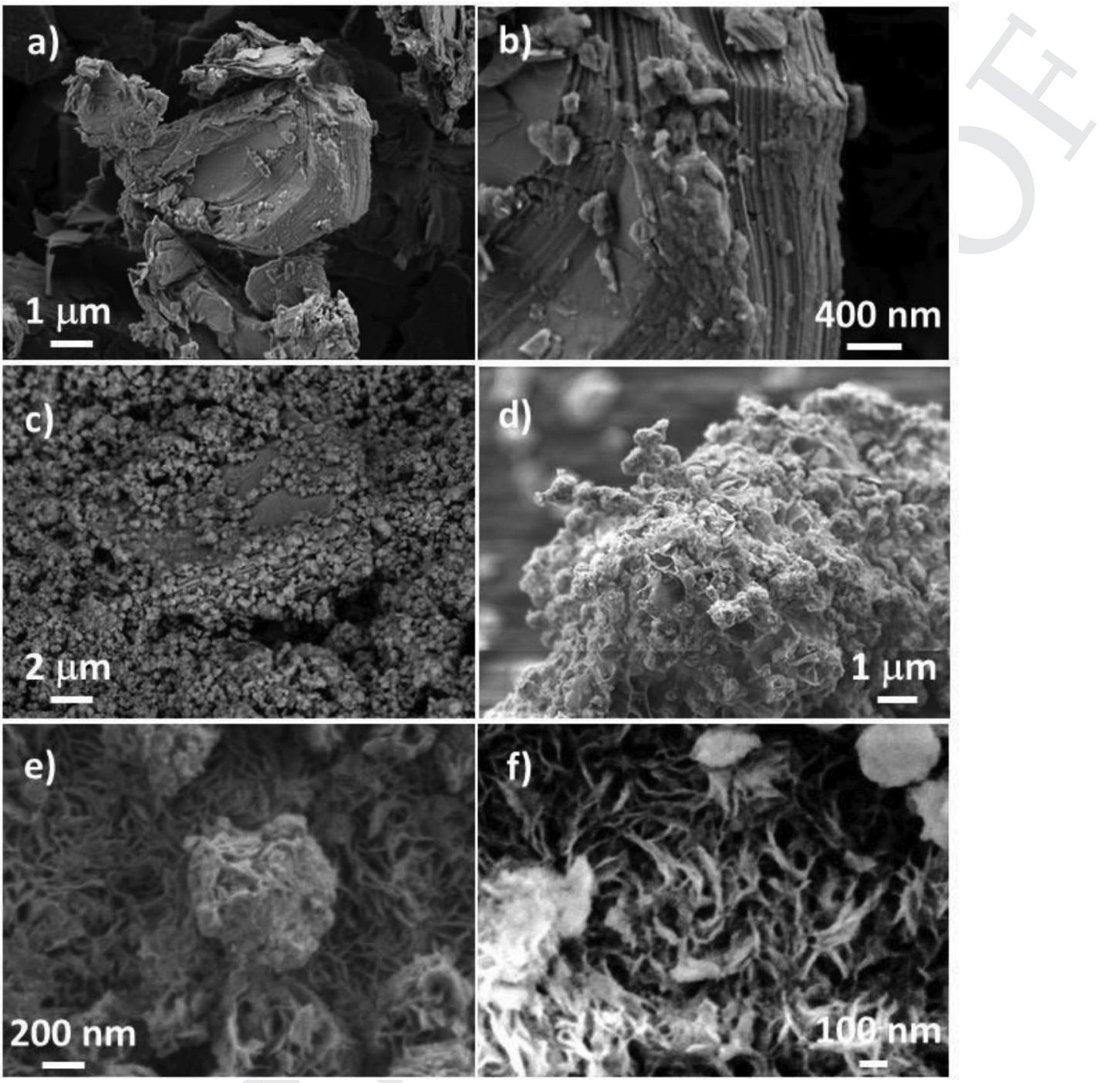

Figure 3. SEM micrographs of a,b) $\mathrm{Ti}_{3} \mathrm{C}_{2} \mathrm{~T}_{x}$ and c-f) Co-LDH@ $@ \mathrm{Ti}_{3} \mathrm{C}_{2} \mathrm{~T}_{x}$ at different magnifications.

characteristic of a more disturbed MXene stack evidenced by additional diffraction spots between the main ones. However, the EFED pattern reveals the absence of oxide on the sample, evidencing the chemical stability of the MXene despite the disorder. The degree of disorder in the MXene stack is apparently closely related to the Co phase coverage, which is inhomogeneous at the nanoscale: increasing the cobalt deposition tends to perturb the MXene stack organization, most probably due to Co intercalation in between the MXene sheets as evidenced later by EELS.

The Co-LDH coverage induces a modification of the MXene's electronic structure as evidenced using EELS, and characteristic of the interaction between Co-LDH and MXene. As an illustration, Figure $7 \mathrm{a}-\mathrm{c}$ shows the EELS spectra collected on the different zones discussed in Figure 6 and their comparison with data collected on reference $\mathrm{Ti}_{3} \mathrm{C}_{2} \mathrm{~T}_{x}$ flakes. The low loss spectra recorded on zones 1a and 2 (Figure 7a), characteristic of the excitation of the valence electron gas in the MXene stack, are typical of MXenes: both the positions and global shapes of

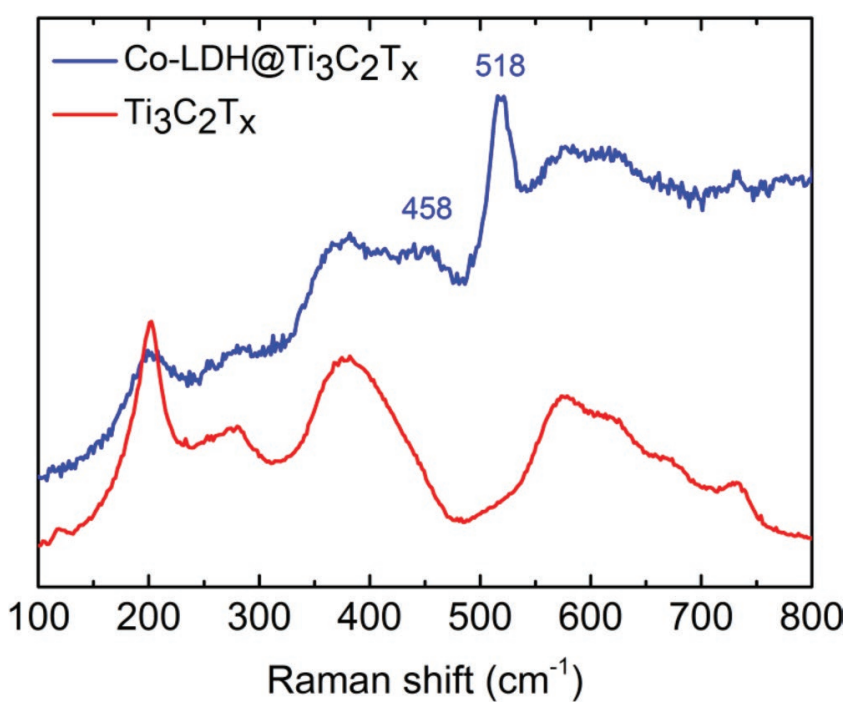

Figure 4. Raman spectra of $\mathrm{Ti}_{3} \mathrm{C}_{2} \mathrm{~T}_{x}$ and Co-LDH@ $@ \mathrm{Ti}_{3} \mathrm{C}_{2} \mathrm{~T}_{x}$ composite. 

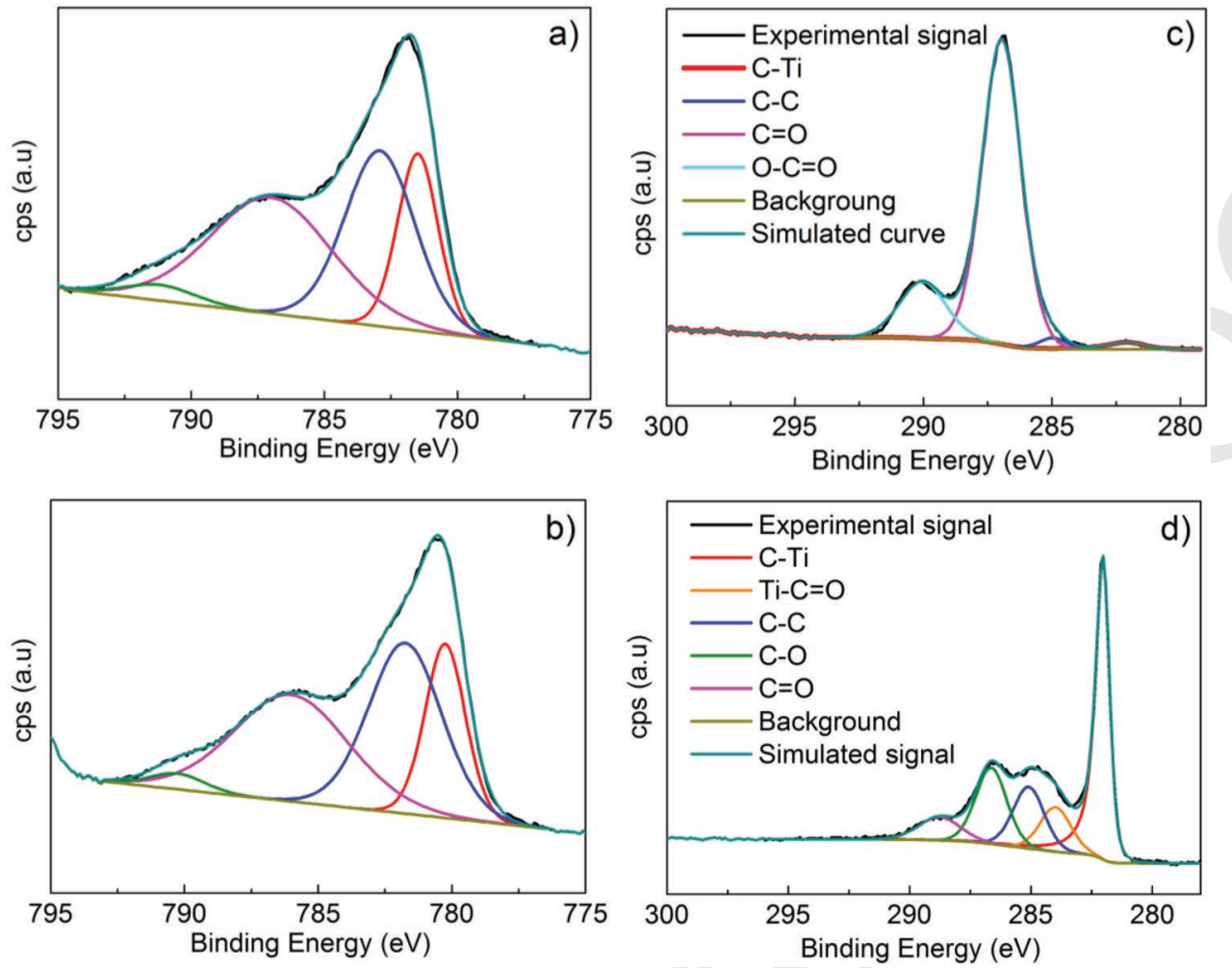

Figure 5. High resolution XPS spectra: Co 2p for a) Co-LDH@ @i $i_{3} \mathrm{C}_{2} \mathrm{~T}_{x}$ and b) commercial Co(OH) ${ }_{2}$ and $\mathrm{C}$ 1s for c) Co-LDH@ $@ \mathrm{Ti}_{3} \mathrm{C}_{2} \mathrm{~T}_{x}$ and d) $\mathrm{Ti}_{3} \mathrm{C}_{2} \mathrm{~T}_{x}$.

the bulk plasmon (labeled C), and Ti $\mathrm{M}_{2,3}$ edge (labeled D and E) are in agreement with the reference spectrum (top curve), ${ }^{[25]}$ confirming the preservation of the MXene general integrity. Very importantly, one can notice the structure F corresponding to the $\mathrm{Co}_{2,3}$ edge attesting for the presence of Co on these MXene sheets. This was confirmed by the recording of the Co $\mathrm{L}_{2,3}$ edge (not shown here) on each one of these zones.

The $\mathrm{C}-\mathrm{K}$ and $\mathrm{Ti} \mathrm{L}_{2,3}$ edges (providing element-selective information) were also recorded in order to characterize the electronic structure and local order within the MXene sheets. The C-K edges recorded on the different zones are presented in Figure 7b. The spectra confirm the analysis of the low-loss region: the spectrum collected in zone $1 \mathrm{a}$ is quite similar to
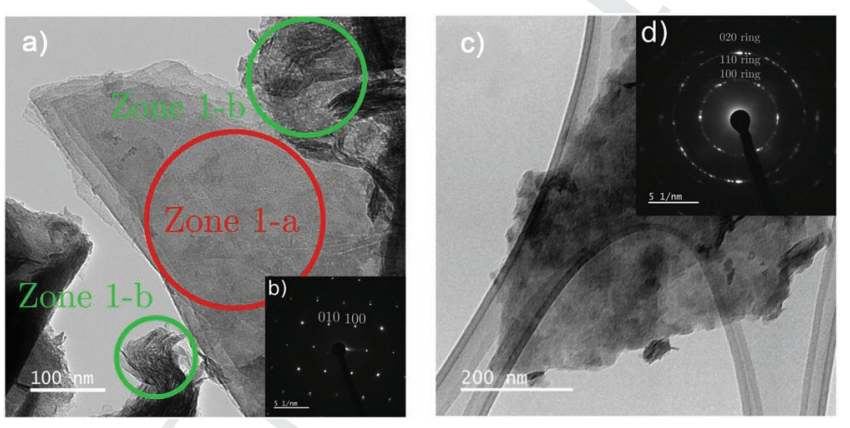

Figure 6. a) Micrography evidencing the coexistence of a clean stack of $\mathrm{Ti}_{3} \mathrm{C}_{2} \mathrm{~T}_{x}$ sheets labeled zone 1-a (see the corresponding EFEDP in b)) and the fibrous Co-LDH in zones 1-b. c) Micrography of zone 2 evidencing a more disturbed MXene stack due to higher Co coverage (see the corresponding EFEDP in d)). the reference spectrum. ${ }^{[2]}$ All the peaks A to D are present at the same energy positions (including the shoulder $\mathrm{B}^{\prime}$ of structure B) with rather similar relative intensities with the noticeable exception of structure A, which has a significantly lower intensity. Part of this structure A was shown from DFT calculations to result from the hybridization between the unoccupied C-p and surface Ti-d states in the $\mathrm{Ti}_{3} \mathrm{C}_{2} \mathrm{~T}_{2}$ system ${ }^{[26]}$ suggesting a modification of the surface chemistry of the MXene support during the deposition of Co-LDH phase. This point is herein confirmed by DFT calculations performed on various structural models with different surface chemistries.

For the here-presented calculations, a $2 \times 2 \times 1$ supercell consisting of two MXene sheets was used with nominal composition $\mathrm{Ti}_{3} \mathrm{C}_{2} \mathrm{~F}_{x}$ (as evidenced by Magne et al. ${ }^{[26]}-\mathrm{F}$ and $-\mathrm{OH}$ terminated systems have very similar $\mathrm{C}-\mathrm{K}$ edges).

Since this structural model generates four surfaces, four different surface configurations were considered (see Figure 8a): one surface fully covered ( $4 \mathrm{~F}$ atoms in $2 \times 2 \times 1$ supercelllabeled F4 in Figure 8a), one surface completely uncovered (labeled F0 in Figure 8a), one surface covered with $3 \mathrm{~F}$ atoms (labeled F3 in Figure 8a), and the last one covered with 1F atom (labeled F1 in Figure 8a). The K-edges corresponding to the carbon atoms below each one of these surfaces have then been simulated and are given in Figure 8b. These calculations all evidence the high sensitivity of the peak A to the surface functionalization, most of the investigated defects resulting in the decrease of the structure $\mathrm{A}$ intensity as observed in the case of the Co-LDH@ $@ \mathrm{Ti}_{3} \mathrm{C}_{2} \mathrm{~T}_{x}$ composite. In addition, our calculations also show the almost complete insensitivity of the structures at higher energy (from B to D). The high energy 

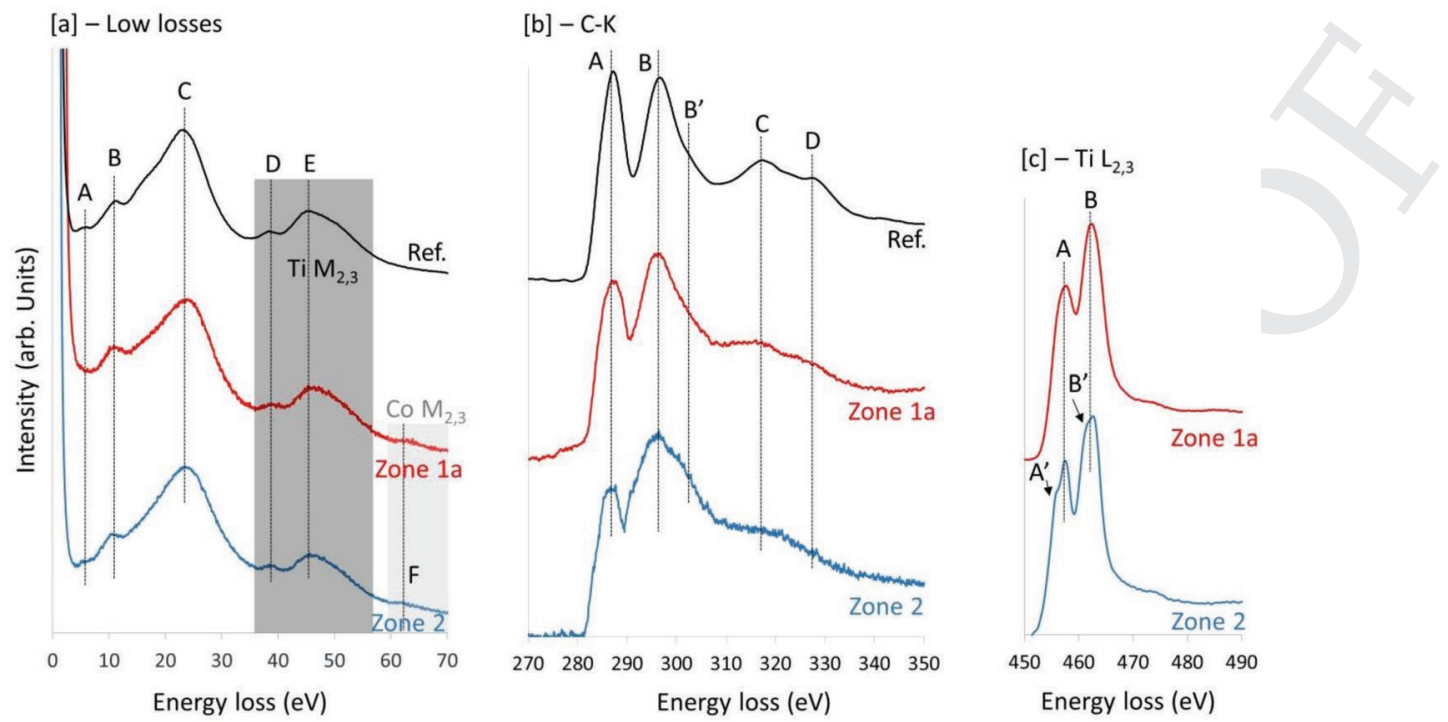

Figure 7. a) Low loss spectra recorded on the zones 1-a (red) and 2 (blue) of the figure. b) C-K and c) Ti $\mathrm{L}_{2,3}$ edges recorded on zones 1-a and 2. Black curves correspond to reference spectra obtained on a pure $\mathrm{Ti}_{3} \mathrm{C}_{2} \mathrm{~T}_{x}$ sample synthesized using the $\mathrm{LiF} / \mathrm{HCl}$ etching method.

region is thus much more characteristic of the inner electronic structure of the MXene sheet in agreement with the analysis of Magne et al. ${ }^{[26]}$ These results suggest that although the MXene sheets have been preserved, their surface chemistry/electronic structure is modified upon functionalization with the Co nanoparticles. Very importantly, this effect is observed at the entire flake scale, evidencing the global coupling between the catalyst and its support. One can notice that in the spectrum recorded in zone 2 (Figure 7b), the structures B, C, and D are broadened evidencing a more pronounced effect on the electronic structure of the inner part of the MXene sheet. These data complement the corresponding EFEDP (Figure 7d) evidencing probably more defects within the corr It is important to notice that defects may play a positive role in 25 the present composite serving as anchoring points for the cata- 26 lyst dispersion. Another important point is that these defects do 27 not alter the general valence band structure of the material as 28 probed in the low loss region and discussed before (see the low 29 loss spectra in Figure 7a).

To complete the EELS analysis, titanium edges were 31 recorded. The Ti $\mathrm{L}_{2,3}$ edge of zone 1 on Figure $7 \mathrm{c}$ ) is charac- 32 teristic of $\mathrm{Ti}_{3} \mathrm{C}_{2} \mathrm{~T}_{x}$. Hence, in agreement with the EFDP, spec- 33 troscopy (LL, C-K, and $\mathrm{Ti} \mathrm{L}_{2,3}$ edges) confirms that the inner 34 structure of the $\mathrm{Ti}_{3} \mathrm{C}_{2} \mathrm{~T}_{x}$ sheets is preserved. The $\mathrm{Ti} \mathrm{L}_{2,3}$ edge 35

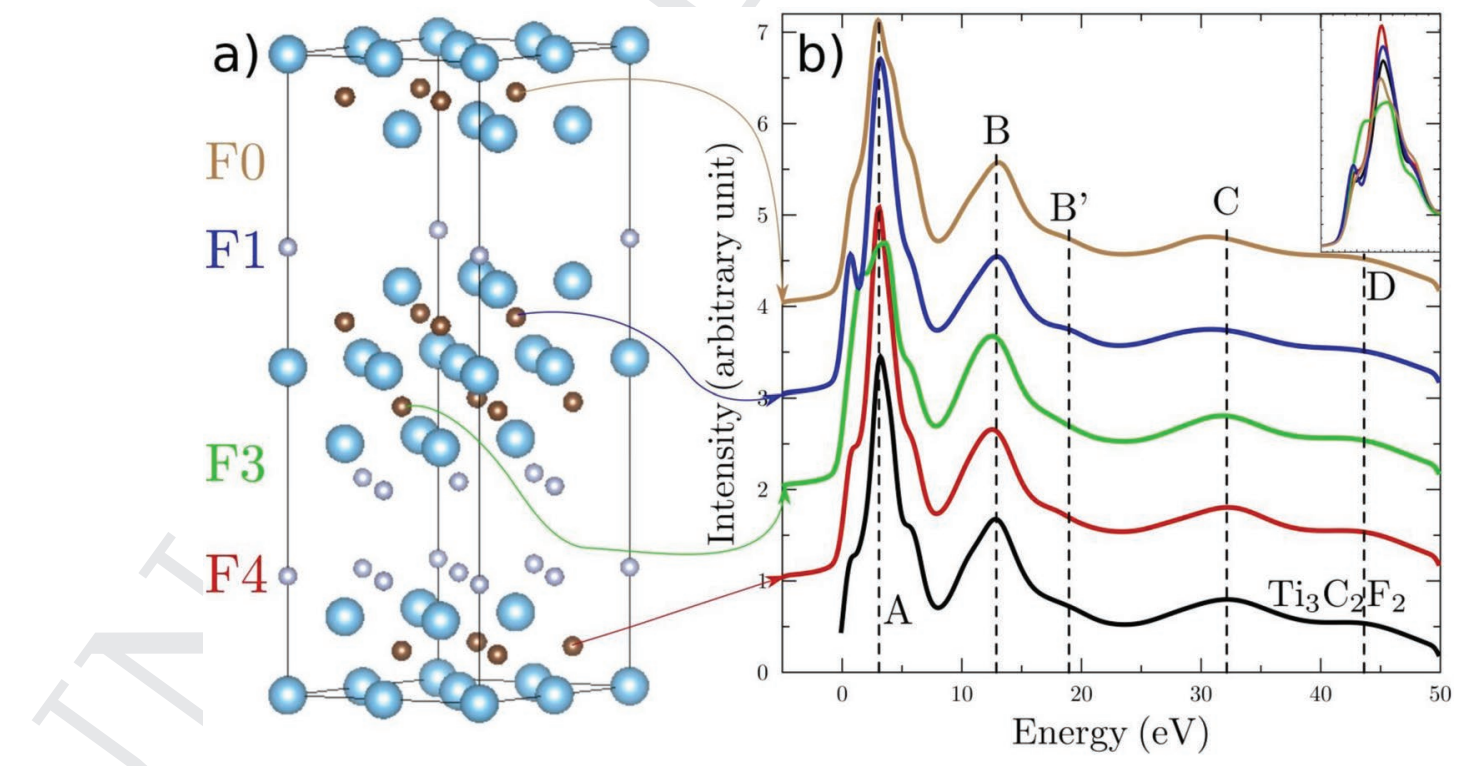

Figure 8. a) $2 \times 2 \times 1$ supercell considered for the simulations and showing the four different surface terminations. Carbon atoms: small brown spheres, titanium atoms: large blue spheres, and fluorine atoms: small gray spheres. b) Calculated fine structure at the $C$ K-edges for the different carbon atoms under each one of the surfaces. Inset: peak A, highly dependent on the surface chemistry. The energy is given with respect to the Fermi energy. 

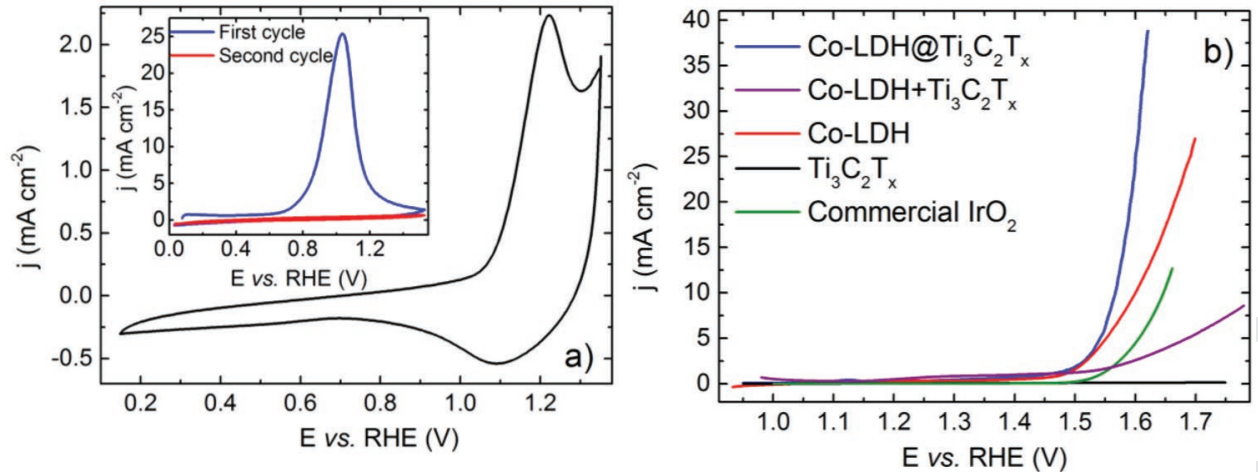

Figure 9. a) Voltammograms recorded in a nitrogen-saturated $1 \mathrm{~mol} \mathrm{~L}-1 \mathrm{KOH}$ electrolyte with $\mathrm{Ti}_{3} \mathrm{C}_{2} \mathrm{~T}_{x}$ sample (inset graph) and with Co-LDH@Ti $\mathrm{C}_{2} \mathrm{~T}_{x}$ catalyst. Scan rate $50 \mathrm{mV} \mathrm{s}^{-1}$. b) Linear sweep voltammograms recorded in a nitrogen-saturated $1 \mathrm{~mol} \mathrm{~L} \mathrm{~L}^{-1} \mathrm{KOH}$ electrolyte with $\mathrm{Ti}_{3} \mathrm{C}_{2} \mathrm{~T}_{x}$ sample, Co-LDH, Co-LDH@Ti ${ }_{3} C_{2} T_{x}$ Co-LDH $+\mathrm{Ti}_{3} \mathrm{C}_{2} \mathrm{~T}_{x}$ and commercial IrO $\mathrm{O}_{2}$ catalysts using a rotating disk electrode set at $1600 \mathrm{rpm}$. Co-LDH $+\mathrm{Ti}_{3} \mathrm{C}_{2} \mathrm{~T}_{x}$ refers to a sample resulting from the mechanical mixing of $\mathrm{Co}-\mathrm{LDH}$ and $\mathrm{Ti}_{3} \mathrm{C}_{2} \mathrm{~T}_{x}$ materials. Scan rate $5 \mathrm{mV} \mathrm{s}^{-1}$.

acquired on zone 2 present a slight splitting of both the $L_{3}$ and $\mathrm{L}_{2}$ edges (labeled $\mathrm{A}^{\prime}$ and $\mathrm{B}^{\prime}$ respectively), which indicate a modification of the electronic structure of the material. As already mentioned, this is supported by the broadening of structure B, $\mathrm{C}$, and D at the C-K edge. However, the structure of the MXene is conserved according to diffraction and these modifications do not result from the oxidation of the sheets.

From this analysis, it turns out that the covering of the MXene with the cobalt phase results in the conservation of the structure of the material and in a strong coupling between the Co-LDH catalyst and the MXene support as revealed by EELS. We propose these electronic structure modifications to be characteristic of the synergistic effect between the MXene and Co-LDH phase explaining the high electrocatalytic performances of this composite as described in the next section.

\subsection{Electrochemical Performances}

The electrochemical behavior of Co-LDH@ $\mathrm{Ti}_{3} \mathrm{C}_{2} \mathrm{~T}_{x}$ and $\mathrm{Ti}_{3} \mathrm{C}_{2} \mathrm{~T}_{x}$ materials was first characterized in a nitrogen-saturated $1 \mathrm{~mol} \mathrm{~L}^{-1} \mathrm{KOH}$ electrolyte using a scan rate of $50 \mathrm{mV} \mathrm{s}^{-1}$. The resulting cyclic voltammograms are presented in Figure 9a.

For the $\mathrm{Ti}_{3} \mathrm{C}_{2} \mathrm{~T}_{x}$ sample, an irreversible oxidation peak centered at $\approx 0.92 \mathrm{~V}$ versus $\mathrm{RH}[\mathrm{I}$ : observed during the positive going scan of the first volt......etric cycle. In the absence of redox active species in the electrolyte, this redox peak is assigned to the oxidation of the $\mathrm{Ti}_{3} \mathrm{C}_{2} \mathrm{~T}_{x}$ material and especially to the oxidation of $\mathrm{Ti}$ atoms. ${ }^{[19]}$ After the first voltammetric cycle, no further surface oxidation can be observed during the second cycle as evidenced by the disappearance of the irreversible oxidation peak centered at $0.92 \mathrm{~V}$ (inset graph of Figure 9a and Figure S5, Supporting Information). This shows that the surface of $\mathrm{Ti}_{3} \mathrm{C}_{2} \mathrm{~T}_{x}$ is then fully oxidized. The deposition of the Co-LDH phase onto the MXene surface appears to prevent the surface oxidation of the MXene as evidenced by the disappearance of the irreversible oxidation peak during the first voltammetric cycle recorded for the composite material (Figure 9a). Taking into account results obtained from physico-chemical characterization of Co-LDH@ $\mathrm{Ti}_{3} \mathrm{C}_{2} \mathrm{~T}_{x}$ composite showing that surface chemistry of $\mathrm{Ti}_{3} \mathrm{C}_{2} \mathrm{~T}_{x}$ is preserved consequently to Co-LDH deposition, it can be deduced that Co-LDH phase acts as a protective layer against MXene oxidation and makes possible the presence of nonoxidized MXenes at electrode potentials required for the OER. This is of upmost importance in order to preserve a high electronic conductivity and decrease the charge transfer resistance at the interface between Co-LDH and $\mathrm{Ti}_{3} \mathrm{C}_{2} \mathrm{~T}_{x}$. This constitutes a major result of the present study.

Taking into account thermodynamic data provided by Meier et $\mathrm{al}^{\left[{ }^{[27]}\right.}$ one can assume that the large oxidation peak centered at $\approx 1.2 \mathrm{~V}$ versus $\mathrm{RHE}$ for the composite electrode during the positive scan can be ascribed to $\mathrm{Co}^{2+} / \mathrm{Co}^{3+}$ redox transition. At higher potentials (i.e., higher than $1.3 \mathrm{~V}$ vs RHE), $\mathrm{Co}^{3+}$ containing surface species are oxidized into $\mathrm{Co}^{4+}$ giving rise to the formation of active sites for OER. ${ }^{[4]}$ The electroactivity of the as-prepared composite toward OER was evaluated by recording linear sweep voltammograms in a nitrogen-saturated $1 \mathrm{~mol} \mathrm{~L}^{-1} \mathrm{KOH}$ electrolyte at a scan rate of $5 \mathrm{mV} \mathrm{s}^{-1}$ and using a rotating disk electrode set at a rotating rate of $1600 \mathrm{rpm}$. The ohmic drop corrected curves presented in Figure 9b first indicate the high activity of Co-LDH@ $\mathrm{Ti}_{3} \mathrm{C}_{2} \mathrm{~T}_{x}$ composite catalyst toward OER since the electrode potential required to drive a current density of $10 \mathrm{~mA} \mathrm{~cm}{ }^{-2}\left(E_{j 10}\right)$ is only of $1.57 \mathrm{~V}$ versus RHE and much lower than the one obtained for unsupported Co-LDH (1.62 V vs RHE). This underlines the positive role played by the intimate contact between $\mathrm{Ti}_{3} \mathrm{C}_{2} \mathrm{~T}_{x}$ and Co-LDH. Note that the amount of Co species, considered as active nanocatalysts, is lower in the composite. Performances of Co-LDH+Ti ${ }_{3} \mathrm{C}_{2} \mathrm{~T}_{x}$ sample resulting from the mechanical mixing of Co-LDH and $\mathrm{Ti}_{3} \mathrm{C}_{2} \mathrm{~T}_{x}$ powders are also lower than for Co-LDH@ $\mathrm{Ti}_{3} \mathrm{C}_{2} \mathrm{~T}_{x}$ material. This can be either due to a higher charge transfer resistance resulting from the low intimacy of the contact between both phases and to the low phase purity of supportless synthesized Co-LDH material as discussed in the manuscript (Figure 1c). The lower activity of the mixture in comparison with Co-LDH alone can be explained by a lower amount of active site (amount of cobalt). Furthermore the electrochemical performance obtained herein with Co-LDH@ $\mathrm{Ti}_{3} \mathrm{C}_{2} \mathrm{~T}_{x}$ composite catalyst are better than those obtained with the $\mathrm{IrO}_{2}$ commercial catalyst $\left(E_{\mathrm{j} 10}=1.64 \mathrm{~V}\right.$ vs RHE) and of the same order of magnitude as those reported for other 
MXene-supported LDH materials (see Table S1 in the Supporting Information) with an easier synthesis route allowing preserving the MXene surface state. The Tafel slope values obtained are another parameter confirming the high efficiency of the Co-LDH@ $\mathrm{Ti}_{3} \mathrm{C}_{2} \mathrm{~T}_{x}$ composite catalyst toward OER. This latter catalyst indeed exhibits a Tafel slope of $82 \mathrm{mV} \mathrm{\textrm {dec } ^ { - 1 }}$ whereas it is of $98 \mathrm{mV} \mathrm{dec}^{-1}$ for the unsupported Co-LDH material. Finally as shown in Figure S6 (Supporting Information), this catalyst is capable of delivering a constant operating potential value of 1.52 and $1.56 \mathrm{~V}$ versus RHE for $20 \mathrm{~h}$ when galvanostatically biased at 5 and $10 \mathrm{~mA} \mathrm{~cm}^{-2}$, respectively.

\section{Conclusion}

In summary, a new and simple polyol method is here proposed for the synthesis of Co-LDH@MXene composite showing high performances for oxygen evolution reaction. The presence of the layered MXene favors the growth of this Co-based structure, highly active for the studied reaction. The presence of terminal groups on the MXene structure allows a good dispersion of the active phase. By this way, the MXene structure can be preserved from oxidation during the composite synthesis as well as under high potential during the electrochemical analyses by total recovery of its surface with the cobaltite phase. This constitutes a major result of this study and, more generally, for applications involving MXenes as support (batteries, supercapacitors, etc.). Indeed, the preservation of the MXene structure is crucial to maintain high electrical conductivity, a key factor to perform efficient electrodes for metal-air batteries or water electrolyzers. It has also been shown by EELS in an original way that high interaction appears between Co-LDH and the MXene with the modification of the MXene surface electronic structure, probably thanks to the presence of terminal groups on the MXene surface. This interaction is a key element allowing to improve the electrochemical performances in comparison with the cobaltite phase alone through a synergistic effect.

Considering the simplicity of the synthesis method and the possibility to easily tune both the nature of the active phase and the MXene composition, there is little doubt that there is room for major improvements in the near future for the design of much more efficient MXene-based oxygen electrocatalysts with major impact for energy storage and conversion applications.

\section{Experimental Section}

Synthesis of $\mathrm{Ti}_{3} \mathrm{C}_{2} \mathrm{~T}_{x}$ : The synthesis of $\mathrm{Ti}_{3} \mathrm{C}_{2} \mathrm{~T}_{x}$ materials is based on the previous work. ${ }^{[12 \mathrm{~b}]} 1 \mathrm{~g}$ of $\mathrm{LiF}$ (Aldrich) were added to $20 \mathrm{~mL}$ of $\mathrm{HCl}$ $6 \mathrm{~mol} \mathrm{~L}^{-1}$, prepared from $\mathrm{HCl} 37 \%$ (Sigma-Aldrich) and stirred during $0.5 \mathrm{~h}$. Then, $1 \mathrm{~g}$ of $\mathrm{Ti}_{3} \mathrm{AlC}_{2}$ (see part I in the Supporting Information for the MAX synthesis) with initial particle size lower than $25 \mu \mathrm{m}$ obtained after sieving, were progressively introduced in the solution to avoid initial overheating. The mixture was heated at $60{ }^{\circ} \mathrm{C}$ for $72 \mathrm{~h}$ and maintained under magnetic stirring. Then, the suspension was washed and centrifuged 4 times with water at $6000 \mathrm{rpm}$ for $6 \mathrm{~min}$, the supernatant liquid being decanted each time. The sediment was filtered using a vacuum-assisted filtration device (PVDF membrane, $0.22 \mu \mathrm{m}$ pore size) and dried overnight at $80^{\circ} \mathrm{C}$.

Synthesis of Co-LDH@ $@ \mathrm{Ti}_{3} \mathrm{C}_{2} \mathrm{~T}_{x}$ and $\mathrm{Co}-\mathrm{LDH}$ : In order to synthesize the Co-LDH@ $\mathrm{Ti}_{3} \mathrm{C}_{2} \mathrm{~T}_{x}$ composite, $190 \mathrm{mg}$ of $\mathrm{Ti}_{3} \mathrm{C}_{2} \mathrm{~T}_{x}$ powder was dispersed first in $196 \mathrm{~mL}$ of ethylene glycol (99.8\%, Sigma Aldrich) 1 with an ultrasound bath for $10 \mathrm{~min}$ under argon, Ar, atmosphere to 2 delaminate the MXene sheets. Then, $570 \mathrm{mg}$ of anhydrous cobalt (II) 3 acetate $(98+\%$, Alfa Aesar) was added to this suspension. After $0.5 \mathrm{~h}$, $130 \mathrm{mg}$ of $\mathrm{NaOH}(\geq 97 \%$, Aldrich) were added and the mixture was 4 stirred under Ar overnight at room temperature, RT. Then, the solution 5 was transferred into a sealed autoclave before being heated at $200{ }^{\circ} \mathrm{C} \quad 6$ for $3 \mathrm{~h}$ under $\mathrm{N}_{2}$ flow. The final product was centrifuged at $9000 \mathrm{rpm} 7$ for 15 min after being dispersed in ethanol (1st washing) and deionized 8 water (2nd washing). The supernatant liquid was decanted and the 9 sediment was filtered, washed with deionized water and dried overnight at RT. Assuming the initial composition of the MXene is $\mathrm{Ti}_{3} \mathrm{C}_{2}(\mathrm{OH})_{2}$, the amount of each precursor was selected to obtain a final ratio of 3.6 cobalt atom per $\mathrm{Ti}_{3} \mathrm{C}_{2}(\mathrm{OH})_{2}$ unit corresponding to a $\mathrm{Co} / \mathrm{Ti}$ atomic ratio of 1.2. 12 The amount of Co was chosen to obtain a high coverage of the MXene.

The sample labeled Co-LDH was produced in the same way, but without the addition of MXene powder.

Material Characterization: The morphology of the synthesized composite was characterized using a field emission gun scanning electron microscope (FEG-SEM) 7900F from JEOL. This microscope is equipped with an energy dispersive X-ray spectrometer (EDS) from Brücker (with Esprit software) allowing the mapping of the $\mathrm{Ti}$ and $\mathrm{Co}$ elements.

XRD patterns were recorded using PANalytical EMPYREAN powder diffractometer using Co $K_{\alpha}$ radiation source $(\lambda=0.1789 \mathrm{~nm})$ in order to limit fluorescence phenomenon. Diffractograms were collected between $5^{\circ}$ and $80^{\circ}$ applying a step size of $0.066^{\circ}$ and a step time of $420 \mathrm{~s}$.

The composites were also examined by Raman spectroscopy. These experiments were carried out using a HORIBA Jobin Yvon HR800 confocal Raman microscope with a CCD detector. Spectra were acquired at RT using an excitation wavelength of $632.8 \mathrm{~nm}$.

The $\mathrm{Al}, \mathrm{Ti}$, and $\mathrm{Co}$ contents of the composite were determined by inductively coupled plasma-optical emission spectrometry using a PerkinElmer Optima 2000DV instrument at a base pressure of $9 \times 10^{-8} \mathrm{~Pa}$.

The XPS analysis were carried out with a Kratos Axis Ultra DLD spectrometer using a monochromatic Al $\mathrm{K}_{\alpha}$ source $(1486 \mathrm{eV}, 10 \mathrm{~mA}$, $15 \mathrm{kV})$. When required, a charge neutralizer system was operated for the analysis. Instrument base pressure was $9 \times 10^{-8} \mathrm{~Pa}$. High-resolution spectra were recorded using an analysis area of $300 \mu \mathrm{m} \times 700 \mu \mathrm{m}^{2}$ and a $20 \mathrm{eV}$ pass energy. These pass energies correspond to $\mathrm{Ag} 3 \mathrm{~d}_{5 / 2}$ FWHM of $0.55 \mathrm{eV}$. Data were acquired with $0.1 \mathrm{eV}$ steps. All the binding energies were calibrated with the $\mathrm{Cls}\left(\mathrm{C}-\mathrm{Ti}_{\mathrm{i}} \mathrm{T}_{x}\right)$ binding energy fixed at $282 \mathrm{eV}$ as an internal reference.

Transmission electron microscopy experiments were performed on reference $\mathrm{Ti}_{3} \mathrm{C}_{2} \mathrm{~T}_{x}$ samples as well as on the Co-LDH@Ti ${ }_{3} \mathrm{C}_{2} \mathrm{~T}_{x}$ composites obtained using the polyol method. Samples were characterized using electron energy loss spectroscopy and energy filtered electron diffraction in order to check the MXene structure both at the short range level (fine structure at core edges in EELS being sensitive to the local order on $a \approx 6-7 \AA$ scale around the excited atom ${ }^{[28]}$ ) and long range level. In addition, TEM-EELS and EFED were also used to characterize the nature of the obtained $\mathrm{Co}$ active phase. Experiments were performed in a JEOL 2200 FS microscope equipped with an in-column omega filter. The operating voltage of the TEM was $200 \mathrm{kV}$, the energy resolution in EELS was about $1.0 \mathrm{eV}$ and the collection angle was fixed to $7 \mathrm{mRad}$. EEL spectra were recorded in the core-loss region, giving access to the electronic structure (the unoccupied $\mathrm{p}$-states for $\mathrm{K}$ edges) projected on the excited atoms, and in the low-loss region which corresponds to the excitation of valence electrons. Core-loss spectra were extracted from the background using a power law and the multiple scattering was removed using the well-known Fourier-ratio method. ${ }^{[29]}$ EFED patterns (EFEDP) were acquired using a $10 \mathrm{eV}$ slit and analyzed using the software JEMS. ${ }^{[30]}$ The TEM samples were prepared by dispersing the composite powders on the inner surface of a beaker and then picking them up on a TEM copper grid covered with lacy carbon film.

Density Functional Theory Calculations: The energy loss near edge 58 structure (ELNES) at the C K-edge and its connection to the surface 18 26 . (2) 年 34 35 35 37 .

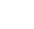
4 . . 列 13

\section{6} (1) 19 (16 
functionalization of the MXene is interpreted by comparison with simulations based on the density functional theory, DFT, and performed with the WIEN2K code. ${ }^{[3]]}$ WIEN2K is an all-electron code using the full-potential augmented plane wave plus local orbitals method to solve the Kohn-Sham equations. Calculations were performed in the generalized gradient approximation using the PBE exchange-correlation functional, ${ }^{[32]}$ which has been shown to give a good description of EEL spectra in $\mathrm{Ti}_{3} \mathrm{C}_{2} \mathrm{~T}_{x} \cdot{ }^{[25,26]}$

Given that simulations performed on $\mathrm{Ti}_{3} \mathrm{C}_{2} \mathrm{~F}_{2}$ and $\mathrm{Ti}_{3} \mathrm{C}_{2}(\mathrm{OH})_{2}$ were shown to give similar ELNES at the $\mathrm{C}$ K-edge, ${ }^{[26]}$ the herepresented calculations were performed on a $\mathrm{Ti}_{3} \mathrm{C}_{2} \mathrm{~F}_{x}$ system described using a $2 \times 2 \times 1$ supercell ( $P 3 \mathrm{ml}$ space group, $a=b=6.1168 \AA$ and $c=19.58826 \AA$ ) in order to take the core-hole into account and allow for different functionalizations of the MXene sheets as discussed in the text. Atomic sphere (muffin-tin) radii of 1.73, 2.09, and 1.99 a.u. were used 14 for carbon, titanium, and fluoride atoms respectively. The product of the lowest muffin-tin radius with the highest wave vector used in the plane wave expansion $\mathrm{RK}_{\max }$ was converged at 8.0. The first Brillouin zone integration was converged for $1500 \mathrm{k}$-points for the SCF calculation.

The EELS spectra were obtained from the band structure using the 18 Telnes 3 extension of WIEN2k: it allows for the calculation of the electron

inelastic scattering cross-section in a relativistic approach, a very important consideration for anisotropic materials. ${ }^{[33]}$

Electrochemical Measurements: All electrochemical measurements were carried out at RT in a standard three-electrode electrochemical cel using potentiostat Autolab (PGSTAT-302N) coupled with a rotating disc electrode ( $\mathrm{RDE})$. $\mathrm{An} \mathrm{Ag} / \mathrm{AgCl}$ (saturated $\mathrm{KCl}$-filled) $\left(E_{\mathrm{AgCl} / \mathrm{Ag}}=1.02 \mathrm{~V}\right.$ vs $\mathrm{ERH}$ ) electrode and a glassy carbon plate were used, respectively, as a reference and counter electrodes. A $3 \mathrm{~mm}$ diameter glassy carbon (GC) disc was used as the working electrode substrate. The catalytic inks were prepared by dispersing $9 \mathrm{mg}$ of catalyst powder in a mixture composed of ultra-pure water $(500 \mu \mathrm{L})$, isopropanol $(500 \mu \mathrm{L})$, and Nafion $(100 \mu \mathrm{L})$, followed by ultrasonication for $10 \mathrm{~min}$. $3 \mu \mathrm{L}$ of the dispersed catalyst were deposited onto the surface of the working electrode (catalyst loading of $0.35 \mathrm{mg} \mathrm{cm}^{-2}$ ) and the deposit was dried under $\mathrm{N}_{2}$ flow.

The measurements were conducted in a $\mathrm{N}_{2}$ saturated $1 \mathrm{~mol} \mathrm{~L}^{-1}$ $\mathrm{KOH}(86.5 \%, \mathrm{VWR})$ aqueous electrolyte. First, cyclic voltammograms were recorded in a nitrogen-saturated electrolyte from 0.15 to $1.35 \mathrm{~V}$ versus RHE at a scan rate of $50 \mathrm{mV} \mathrm{s}^{-1}$. Then the activity of catalysts toward OER was examined by recording linear sweep voltammograms from 0.95 to $1.75 \mathrm{~V}$ versus $\mathrm{RHE}$ at a scan rate of $5 \mathrm{mV} \mathrm{s}^{-1}$ by applying a rotating rate of $1600 \mathrm{rpm}$ to the RDE. All measurements were IR-drop corrected by determining the cell resistance using electrochemical impedance spectroscopy measurements (EIS) between $0.1 \mathrm{~Hz}-100 \mathrm{KHz}$. Spectra were acquired in the capacitive region with a Solartron SI 1287 electrochemical interface and an SI 1260 impedance/gain-phase analyzer.

These electrochemical measurements were conducted on Co-LDH@ $\mathrm{Ti}_{3} \mathrm{C}_{2} \mathrm{~T}_{x}, \mathrm{Co}-\mathrm{LDH}$, and commercial $\mathrm{IrO}_{2}$ (99.9\%, Alfa Aesar).

\section{Supporting Information}

Supporting Information is available from the Wiley Online Library or from the author.

\section{Acknowledgements}

The authors acknowledge financial support from the "Agence National de la Recherche" (reference ANR-18-CE08-014-MXENECAT project), the European Union (ERDF), the "Région Nouvelle Aquitaine" (Ph.D. thesis of T.B.) and the French research ministry (Ph.D. thesis of M.B.). This work also partially pertains to the French Government program "Investissements d'Avenir" (LABEX INTERACTIFS, reference ANR-11. LABX-0017-01), which is here gratefully acknowledged for the financial support to C.G. post-doctoral position. The authors wish to thank
J. Rousseau for her assistance on SEM analyses. Pr M. Barsoum is highly acknowledged for his valuable advice concerning $1^{\prime \prime}$ '

\section{Conflict of Interest}

The authors declare no conflict of interest.

\section{Keywords}

layered double hydroxide (LDH), MXenes, oxygen evolution reaction, polyol synthesis

Received: July 31, 2019

Revised: September 17, 2019 Published online:

[1] a) M. T. M. Koper, J. Electroanal. Chem. 2011, 660, 254; b) M. W. Kanan, D. G. Nocera, Science 2008, 321, 1072.

[2] a) S. Anantharaj, S. R. Ede, K. Sakthikumar, K. Karthick, S. Mishra, S. Kundu, ACS Catal. 2016, 6, 8069; b) H. N. Nong, T. Reier, H.-S. Oh, M. Gliech, P. Paciok, T. H. T. Vu, D. Teschner, M. Heggen, V. Petkov, R. Schlögl, T. Jones, P. Strasser, Nat. Catal. 2018, 1, 841.

[3] a) S. Cherevko, S. Geiger, O. Kasian, N. Kulyk, J.-P. Grote, A. Savan, B. R. Shrestha, S. Merzlikin, B. Breitbach, A. Ludwig, K. J. J. Mayrhofer, Catal. Today 2016, 262, 170; b) X. Lu, C. Zhao, Nat. Commun. 2015, 6, 6616.

[4] a) I. Abidat, N. Bouchenafa-Saib, A. Habrioux, C. Comminges, C. Canaff, J. Rousseau, T. W. Napporn, D. Dambournet, O. Borkiewicz, K. B. Kokoh, J. Mater. Chem. A 2015, 3, 17433; b) I. Abidat, C. Morais, C. Comminges, C. Canaff, J. Rousseau, N. Guignard, T. W. Napporn, A. Habrioux, K. B. Kokoh, J. Mater. Chem. A 2017, 5, 7173.

[5] a) K. Kumar, C. Canaff, J. Rousseau, S. Arrii-Clacens, T. W. Napporn, A. Habrioux, K. B. Kokoh, J. Phys. Chem. C 2016, 120, 7949; b) K. Kumar, I. Abidat, C. Canaff, A. Habrioux, C. Morais, T. W. Napporn, K. B. Kokoh, ChemElectroChem 2018, 5, 483; c) K. Kumar, L. Loupias, C. Canaff, S. Morisset, S. Pronier, C. Morais, A. Habrioux, T. W. Napporn, K. B. Kokoh, ChemPhysChem 2018, 19, 319.

[6] a) J. Jiang, A. Zhang, L. Li, L. Ai, J. Power Sources 2015, 278, 445; b) H. Lin, Y. Zhang, G. Wang, J.-B. Li, Front. Mater. Sci. 2012, 6, 142; c) X. Xu, Z. Zhong, X. Yan, L. Kang, J. Yao, J. Mater. Chem. A 2018, 6, 5999; d) G. Fan, F. Li, D. G. Evans, X. Duan, Chem. Soc. Rev. 2014, 43, 7040.

[7] a) Z. Jiang, Z. Li, Z. Qin, H. Sun, X. Jiao, D. Chen, Nanoscale 2013 5, 11770; b) H. Hu, B. Guan, B. Xia, X. W. Lou, J. Am. Chem. Soc. 2015, 137, 5590; c) X. Bai, J. Liu, Q. Liu, R. Chen, X. Jing, B. Li, J. Wang, Chem. - Eur. J. 2017, 23, 14839.

[8] a) M. Gong, Y. Li, H. Wang, Y. Liang, J. Z. Wu, J. Zhou, J. Wang, T. Regier, F. Wei, H. Dai, J. Am. Chem. Soc. 2013, 135, 8452; b) M. Yu, S. Zhou, Z. Wang, J. Zhao, J. Qiu, Nano Energy 2018, 44, 181.

[9] M. Alhabeb, K. Maleski, B. Anasori, P. Lelyukh, L. Clark, S. Sin, Y. Gogotsi, Chem. Mater. 2017, 29, 7633.

[10] a) P. Salles, E. Quain, N. Kurra, A. Sarycheva, Y. Gogotsi, Small 2018, 14, 1802864; b) C. Zhang, B. Anasori, A. Seral-Ascaso, S.-H. Park, N. McEvoy, A. Shmeliov, G. S. Duesberg, J. N. Coleman, Y. Gogotsi, V. Nicolosi, Adv. Mater. 2017, 29, 1702678.

[11] M. W. Barsoum, MAX Phases: Properties of Machimaln Ternary Carbides and Nitrides, Wiley-VCH, Weinheim 2013. 
[12] a) M. Naguib, V. N. Mochalin, M. W. Barsoum, Y. Gogotsi, Adv. Mater. 2014, 26, 992; b) M. Chidiu, M. R. Lukatskaya, M.-Q. Zhao, Y. Gogotsi, M. W. Barsoum, Nature 2014, 516, 78; c) X. Wang, C. Garnero, G. Rochard, D. Magne, S. Morisset, S. Hurand, P. Chartier, J. Rousseau, T. Cabioc'h, C. Coutanceau, V. Mauchamp, S. Célérier, J. Mater. Chem. A 2017, 5, 22012.

[13] A. Zehtab Yazdi, E. P. L. Roberts, U. Sundararaj, Carbon 2016, 100, 99.

[14] L. Zhao, B. Dong, S. Li, L. Zhou, L. Lai, Z. Wang, S. Zhao, M. Han, K. Gao, M. Lu, X. Xie, B. Chen, Z. Liu, X. Wang, H. Zhang, H. Li, J. Liu, H. Zhang, X. Huang, W. Huang, ACS Nano 2017, 11, 5800.

[15] H. Zou, B. He, P. Kuang, J. Yu, K. Fan, ACS Appl. Mater. Interfaces 2018, 10, 22311.

[16] L. Xiu, Z. Wang, M. Yu, X. Wu, J. Qiu, ACS Nano 2018, 12, 8017.

[17] Y. Zhang, H. Jiang, Y. Lin, H. Liu, Q. He, C. Wu, T. Duan, L. Song, Adv. Mater. Interfaces 2018, 5, 1800392.

[18] J. Liu, T. Chen, P. Juan, W. Peng, Y. Li, F. Zhang, X. Fan, ChemSusChem 2018, 11, 3758.

[19] P. Nayak, Q. Jiang, R. Mohanraman, D. Anjum, M. N. Hedhili, H. N. Alshareef, Nanoscale 2018, 10, 17030.

[20] X.-D. Zhu, Y. Xie, Y.-T. Liu, J. Mater. Chem. A 2018, 6, 21255.

[21] a) L. Lorencova, T. Bertok, E. Dosekova, A. Holazova, D. Paprckova, A. Vikartovska, V. Sasinkova, J. Filip, P. Kasak, M. Jerigova, D. Velic, K. A. Mahmoud, J. Tkac, Electrochim. Acta 2017, 235, 471; b) L. Lorencova, T. Bertok, J. Filip, M. Jerigova, D. Velic, P. Kasak, K. A. Mahmoud, J. Tkac, Sens. Actuators, B 2018, 263, 360.

[22] S. Célérier, S. Hurand, C. Garnero, S. Morisset, M. Benchakar, A. Habrioux, P. Chartier, V. Mauchamp, N. Findling, B. Lanson, E. Ferrage, Chem. Mater. 2019, 31, 454.
[23] a) V. J. Keast, A. J. Scott, R. Brydson, D. B. Williams, J. Bruley, 1 J. Microsc. 2001, 203, 135; b) J. Yang, T. Regier, J. J. Dynes, J. Wang, 2 J. Shi, D. Peak, Y. Zhao, T. Hu, Y. Chen, J. S. Tse, Anal. Chem. 2011, 3 83, 7856.

[24] a) M. Naguib, O. Mashtalir, M. R. Lukatskaya, B. Dyatkin, C. Zhang, 5 V. Presser, Y. Gogotsi, M. W. Barsoum, Chem. Commun. 2014, 50, 7420; b) C. J. Zhang, S. Pinilla, N. McEvoy, C. P. Cullen, B. Anasori, E. Long, S.-H. Park, A. Seral-Ascaso, A. Shmeliov, D. Krishnan, C. Morant, X. Liu, G. S. Duesberg, Y. Gogotsi, V. Nicolosi, Chem. 8 Mater. 2017, 29, 4848; c) T. Hu, J. Wang, H. Zhang, Z. Li, M. Hu, 9 X. Wang, Phys. Chem. Chem. Phys. 2015, 17, 9997.

[25] D. Magne, V. Mauchamp, S. Célérier, P. Chartier, T. Cabioc'h, Phys. 11 Rev. B 2015, 91, 201409.

[26] D. Magne, V. Mauchamp, S. Célérier, P. Chartier, T. Cabioc'h, Phys. Chem. Chem. Phys. 2016, 18, 30946.

[27] H. G. Meier, J. R. Vilche, A. J. Arvía, J. Electroanal. Chem. Interfacial 14 Electrochem. 1982, 138, 367.

[28] a) M. Bugnet, V. Mauchamp, P. Eklund, M. Jaouen, T. Cabioc'h, Acta Mater. 2013, 61, 7348; b) M. Bugnet, M. Jaouen, V. Mauchamp, T. Cabioc'h, G. Hug, Phys. Rev. B 2014, 90, 195116.

[29] R. F. Egerton, Electron Energy-Loss Spectroscopy in the Electron 19 Microscope, Springer, USA 2011

[30] JEMS website, http://www.jems-saas.ch/ (accessed: 2014).

[31] P. Blaha, K. Schwarz, G. K. H. Madsen, D. Kvasnicka, J. Luitz, An 22 Augmented Plane Wave Plus Local Orbitals Program for Calculating 23 Crystal Properties, University of Technology, Vienna 2001.

[32] J. P. Perdew, K. Burke, M. Ernzerhof, Phys. Rev. Lett. 1996, 77, 3865 $=2$

[33] a) P. Schattschneider, C. Hébert, H. Franco, B. Jouffrey, Phys. Rev. B 26 2005, 72, 045142; b) C. Hébert, Micron 2007, 38, 12. 


\section{Reprint Order Form}

Charges for Reprints in Euro (excl. VAT), prices are subject to change. Minimum order 50 copies.

\begin{tabular}{l|rrrrrr} 
No. of pages & $\begin{array}{c}\mathbf{5 0} \\
\text { copies }\end{array}$ & $\begin{array}{c}\mathbf{1 0 0} \\
\text { copies }\end{array}$ & $\begin{array}{c}\mathbf{1 5 0} \\
\text { copies }\end{array}$ & $\begin{array}{c}\mathbf{2 0 0} \\
\text { copies }\end{array}$ & $\begin{array}{c}\mathbf{3 0 0} \\
\text { copies }\end{array}$ & $\begin{array}{c}\mathbf{5 0 0} \\
\text { copies }\end{array}$ \\
\hline $\mathbf{1 - 4}$ & $345,-$ & $395,-$ & $425,-$ & $445,-$ & $548,-$ & $752,-$ \\
$\mathbf{5 - 8}$ & $490,-$ & $573,-$ & $608,-$ & $636,-$ & $784,-$ & $1077,-$ \\
$\mathbf{9 - 1 2}$ & $640,-$ & $739,-$ & $786,-$ & $824,-$ & $1016,-$ & $1396,-$ \\
$\mathbf{1 3 - 1 6}$ & $780,-$ & $900,-$ & $958,-$ & $1004,-$ & $1237,-$ & $1701,-$ \\
$\mathbf{1 7 - 2 0}$ & $930,-$ & $1070,-$ & $1138,-$ & $1196,-$ & $1489,-$ & $2022,-$ \\
\hline & & & & & & \\
& $147,-$ & $169,-$ & $175,-$ & $188,-$ & $231,-$ & $315,-$ \\
$\begin{array}{l}\text { every additional } \\
\text { 4 pages }\end{array}$ & & & & & &
\end{tabular}

Please send me send bill me for

no. of reprints

high-resolution PDF file (330 Euro excl. VAT)

E-mail address:

* Special Offer:

If you order 200 or more reprints you will get a PDF file for half price.

Please note: It is not permitted to present the PDF file on the internet or on company homepages.

Cover Posters (prices excl. VAT)

Posters of published covers are available in two sizes: DinA2 42 × $60 \mathrm{~cm} / 17$ x 24in (one copy: 39 Euro) DinA1 $60 \times 84 \mathrm{~cm} / 24$ x 33in (one copy: 49 Euro)

Postage for shipping (prices excl. VAT)

overseas +25 Euro

within Europe +15 Euro
Email: advmatinterfaces@wiley-vch.de

Manuscript No.:

Customer No.: (if available)

Purchase Order No.:

Author:

Information regarding VAT: The charges for publication of cover pictures /reprints/issues/poster/Video abstracts/are considered to be "supply of services" and therefore subject to German VAT. However, if you are an institutional customer outside Germany, the tax can be waived if you provide us with the valid VAT number of your company. Non-EU customers may have a VAT number starting with "EU" instead of their country code, if they are registered with the EU tax authorities. If you do not have a valid EU VAT number and you are a taxable person doing business in a non-EU country, please provide a certification from your local tax authorities confirming that you are a taxable person under local tax law. Please note that the certification must confirm that you are a taxable person and are conducting an economic activity in your country. Note: certifications confirming that you are a taxexempt legal body (non-profit organization, public body, school, political party, etc.) in your country do not exempt you from paying German VAT.

VAT number:

Mail reprints / copies of the issue to:

Send bill to:

I will pay by bank transfer

I will pay by credit card

VISA, Mastercard and AMERICAN EXPRESS

For your security please use this link (Credit Card Token Generator) to create a secure code Credit Card Token and include this number in the form instead of the credit card data. Click here: https://www.wiley-vch.de/editorial production/index.php CREDIT CARD TOKEN NUMBER 